\title{
WestVirginiaUniversity
}

THE RESEARCH REPOSITORY @ WVU

Graduate Theses, Dissertations, and Problem Reports

2009

\section{Insomnia symptoms, nightmares and suicidal ideation in a university sample}

\author{
Michael R. Nadorff \\ West Virginia University
}

Follow this and additional works at: https://researchrepository.wvu.edu/etd

\section{Recommended Citation}

Nadorff, Michael R., "Insomnia symptoms, nightmares and suicidal ideation in a university sample" (2009). Graduate Theses, Dissertations, and Problem Reports. 4506.

https://researchrepository.wvu.edu/etd/4506

This Thesis is protected by copyright and/or related rights. It has been brought to you by the The Research Repository @ WVU with permission from the rights-holder(s). You are free to use this Thesis in any way that is permitted by the copyright and related rights legislation that applies to your use. For other uses you must obtain permission from the rights-holder(s) directly, unless additional rights are indicated by a Creative Commons license in the record and/ or on the work itself. This Thesis has been accepted for inclusion in WVU Graduate Theses, Dissertations, and Problem Reports collection by an authorized administrator of The Research Repository @ WVU. For more information, please contact researchrepository@mail.wvu.edu. 
Insomnia Symptoms, Nightmares and Suicidal Ideation in a University Sample

Michael R. Nadorff, B.A.

Thesis submitted to the Eberly College of Arts and Sciences at the West Virginia University

in Partial Fulfillment of the Requirements for the degree of

Master of Science

In

Psychology

Amy Fiske, Ph. D.

Hawley Montgomery-Downs, Ph.D.

Julie Hicks Patrick, Ph.D.

Department of Psychology

Morgantown, WV

2009

Keywords: Suicidal ideation; insomnia symptoms; nightmares

Copyright 2009 Michael R. Nadorff 


\begin{abstract}
Insomnia Symptoms, Nightmares and Suicidal Ideation in a University Sample
\end{abstract}

\title{
Michael Nadorff
}

Previous research has demonstrated a link between insomnia symptoms, nightmares, and suicidal ideation. The purpose of this study was to investigate whether insomnia symptoms and nightmares were related to suicidal ideation independent of each other. Further, the study aimed to determine whether suicidal ideation and nightmares were related with suicidal ideation independent of the symptoms of anxiety, depression, and PTSD. The study consisted of 583 undergraduate students at West Virginia University. Results indicated that both nightmares $(\beta=$ $.054(S E=.008), p>.01)$ and insomnia symptoms $(\beta=.045(S E=.009), p>.01)$ were related to suicidal ideation, and this relation was independent of each other. However, nightmares $(\beta=$ $.021(S E=.008), p=.01)$, but not insomnia symptoms, were related with suicidal ideation independent of the symptoms of anxiety, depression, and PTSD. Implications, limitations and future directions are discussed. 


\section{Acknowledgments}

There are many people I would like to thank for their role in this project. First, I would like to thank my advisor, Amy Fiske, Ph.D. This project would not have been possible without her guidance and support as well as the training I have received by being a member of her lab and one of her advisees. I would also like to extend my thanks to the other members of my committee, Hawley Montgomery-Downs, Ph.D. and Julie Hicks Patrick, Ph.D., for their time, feedback, and guidance throughout the process. Additionally, I would like to thank the members of the Mental Health and Aging Lab for their feedback and support.

I would like to express my appreciation to the members of the psychology department at University of Notre Dame. I would especially like to thank Anita Kelly, Ph.D. for her training and guidance from my first day as a research assistant through my first published article. The experience I gained in her lab has helped me get to where I am today, and hence, has played a vital role in this project.

I would like to thank my friends in the department and my friends from home for their continued support and encouragement. Finally, I would like to thank my family, especially my wife, Danielle Nadorff, for their continued love, support, and encouragement. 
Table of Contents

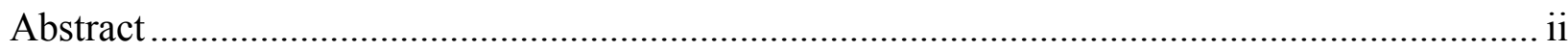

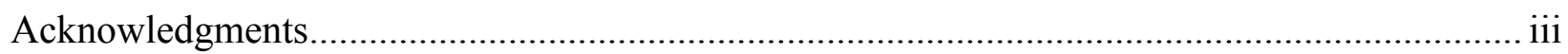

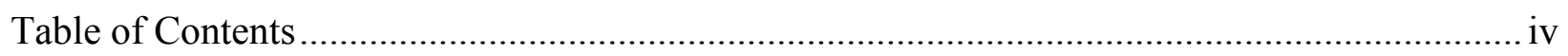

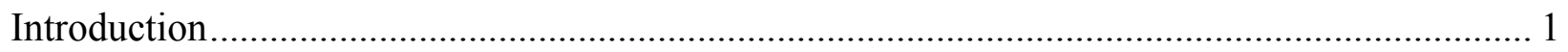

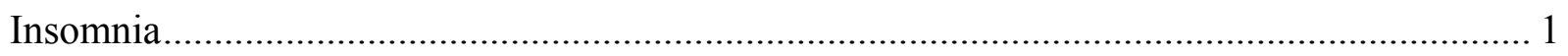

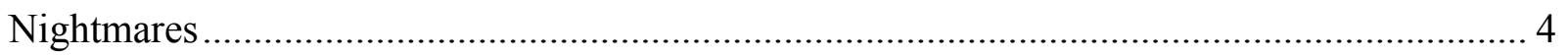

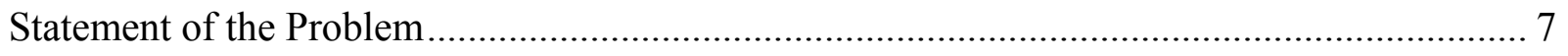

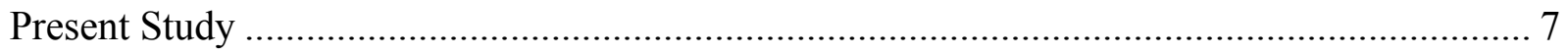

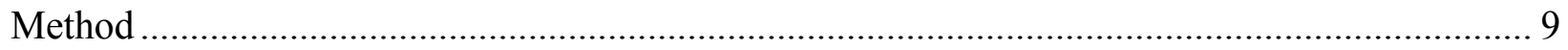

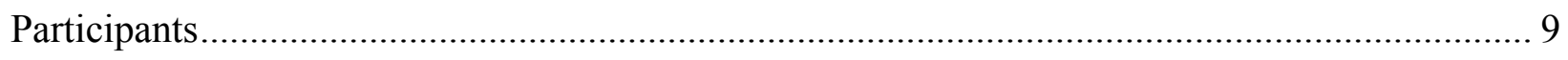

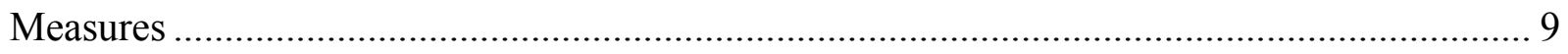

The Disturbing Dreams and Nightmare Severity Index ............................................... 9

The Center of Epidemiological Studies Depression Scale ........................................... 9

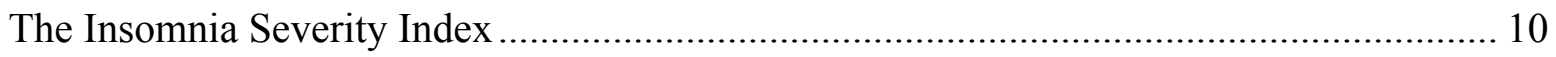

The PTSD Checklist-Civilian Version...................................................................... 10

The Suicidal Behaviors Questionnaire................................................................. 11

The Zung Self-Rating Anxiety Scale ................................................................... 11

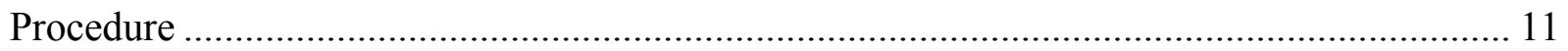

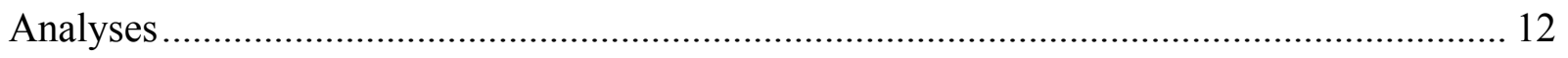

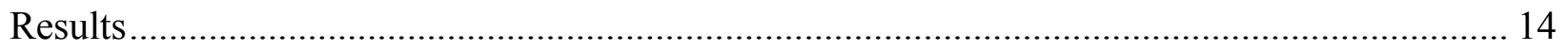

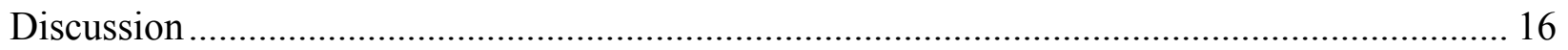




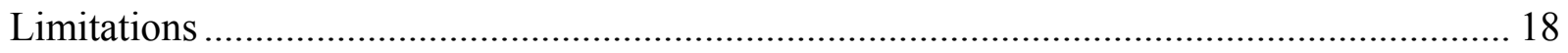

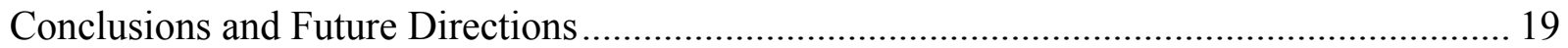

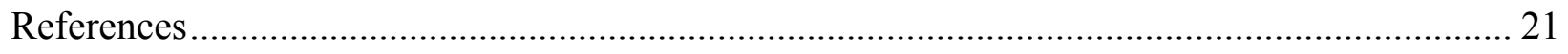

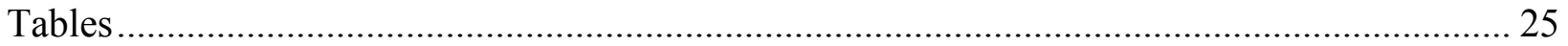

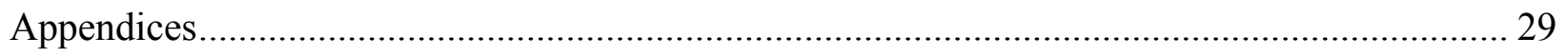


Suicide is a significant problem in the United States. Heron et al. (2009) report that suicide was the $11^{\text {th }}$ leading cause of death in the United States in 2006 accounting for 33,300 deaths. Though suicidal behavior is very complex, steps toward understanding suicidal behavior can be made through the identification of suicide risk factors. Although mental illness has been shown to be a risk-factor for suicide, and over $90 \%$ of those who die by suicide have a mental disorder at the time of their death, the vast majority of people with mental disorders do not die by suicide. Identifying risk factors beyond having a mental disorder may help in determining who is at the highest risk of suicidal behavior. Additionally, designing interventions with these riskfactors in mind may help reduce the risk of suicide in those at high risk of suicidal behavior. Therefore, identifying risk factors for suicide may aid in the identification and treatment of those who are suicidal before they die by suicide.

The current study examined two types of sleep difficulties, insomnia symptoms and nightmares, to test whether they are related to suicidal ideation in a college population and, if so, whether these relations remain after controlling for symptoms of depression, anxiety, and PTSD.

\section{Insomnia}

Insomnia is emerging in the empirical literature as a possible risk factor for suicidal behavior. Barbe et al., (2005) examined children and adolescents aged 7-17 who were either depressed and had suicidal ideation or were depressed without suicidal ideation. The purpose of the research was to determine what comorbid disorders would explain the difference between those with and without suicidal ideation. The Kiddie Schedule for Affective Disorders and Schizophrenia (K-SADS) semi-structured interview was used to assess suicidal ideation and insomnia. Symptoms of insomnia, as assessed by the K-SADS, were significantly more common 
in depressed youth who reported suicidal ideation (72.1\%) than in depressed youth who did not report suicidal ideation $(45.6 \%)$.

Insomnia has also been shown to be related to overt suicidal behavior. Hall, Platt, and Hall (1999) conducted an uncontrolled study of 100 individuals who had made medically serious suicide attempts. The goal of this research was to identify the most frequent correlates in those making serious suicide attempts. The study analyzed two types of insomnia symptoms: global and partial. Global insomnia was defined as endorsing difficulty falling asleep, difficulty staying asleep, and early morning awakenings. Partial insomnia was defined as endorsing difficulty falling asleep, staying asleep, or waking too early but not experiencing all three (R. C. W. Hall, personal communication, May 15, 2008). Hall, Platt, and Hall (1999) found that of those who made medically serious suicide attempts, $64 \%$ qualified for global insomnia and $92 \%$ for partial insomnia. Given that the one-year prevalence of insomnia complaints, which would be similar to partial insomnia, is $30-40 \%$ it seems that a larger proportion of those who attempted suicide experienced insomnia symptoms than in the general population (American Psychiatric Association, 2000). Therefore, this research suggests that symptoms of insomnia are very common in those who seriously attempt suicide, and that insomnia symptoms may be more common than in the general population.

In addition to examining the relation between symptoms of insomnia and suicidal ideation and nonfatal suicide behaviors, several studies have demonstrated a relation between symptoms of insomnia and death by suicide. McGirr et al. (2007) conducted a controlled psychological autopsy study where 156 cases (average age: $42.4, \mathrm{SD}=13.2$ ) who were depressed and died by suicide were compared with 81 major depressive controls (average age: $42.7, \mathrm{SD}=11.1)$. The research aimed to see which symptoms of depression differentiated those 
who died by suicide from the controls. It was found that those who died by suicide were more likely to have had symptoms of insomnia than the depressed controls.

Prospective research has also shown a relation between insomnia and death by suicide. Fujino, Mizoue, Tokui, and Yoshimura (2005) followed 15,597 Japanese adults age 30-79 for 14 years to find which risk factors predicted death by suicide in the general population. In addition, they categorized insomnia symptoms into three different types; onset, maintenance, and terminal. Onset insomnia consists of difficulty falling asleep, maintenance insomnia is difficulty maintaining sleep throughout the night, and terminal insomnia is waking for the last time before the desired wake time. Fujino, et al. (2005) found that insomnia symptoms were a risk factor for dying by suicide independent of age. When broken down by type of insomnia, only symptoms of maintenance insomnia were significantly related to higher risk of dying by suicide. Of those who died by suicide in the sample, 19\% reported onset insomnia, 35\% reported maintenance insomnia, and $25 \%$ reported terminal insomnia at the beginning of the study. One limitation, however, is that they did not report whether insomnia predicted suicide independent of depression.

In another longitudinal study, utilizing a sample of 954 psychiatric patients age 17-79 years (average age 38.1 years) with major affective disorders, Fawcett, Schefner, Fogg, and Clark (1990) aimed to see what clinical features were associated with death by suicide. Global insomnia symptoms, defined as endorsing difficulty falling asleep, difficulty staying asleep, and early morning awakenings, predicted death by suicide within a year.

Thus, evidence shows that insomnia symptoms are related to suicidal ideation, attempts, and death in younger and mixed-age samples. Additionally, among individuals with depression, insomnia symptoms predict death by suicide. However, few of these studies examined whether 
other mental disorders that are often co-morbid with insomnia, such as anxiety and PTSD, explain the relation between suicide and insomnia. Depression and anxiety have been shown to be related to both insomnia symptoms and suicide. Insomnia is often co-morbid with both anxiety and depression, and is listed in the criteria for a diagnosis of depression (American Psychiatric Association, 2000). Recent research has shown that sleep complaints can be characterized by two factors, termed insomnia and lassitude (Koffel \& Watson, 2009). The insomnia factor is highly correlated with anxiety and the lassitude factor correlates with depression. Additionally, both depression and anxiety have been shown to be related to suicide (Tidemalm, Langstrom, Lichtenstein, Runeson, 2008). Given that depression and anxiety are related to both insomnia symptoms and suicidal ideation, it is important to evaluate whether these disorders might explain the relation between them. If insomnia is shown to be independently related to suicidal ideation, it strengthens the argument that insomnia should be considered a risk-factor for suicide.

\section{Nightmares}

Nightmares also appear to be a possible risk factor for suicidal behavior. One possible rationale for studying nightmares in relation to suicidal behavior is that nightmares are related to traumatic events and Posttraumatic Stress Disorder (PTSD), which are in turn associated with suicidal behavior. Wood, Bootzin, Rosenhan, Nolen-Hoeksema, and Jourden, (1992) analyzed differences in nightmares between college students in San Francisco, CA and Tucsan, AZ following the 1989 San Francisco earthquake. They found that those in San Francisco experienced more nightmares than those in Tucsan. This research demonstrates that experiencing a traumatic event is potentially related to having more nightmares. Nightmares have also been shown to be related to PTSD and are part of the criteria for a diagnosis of PTSD (American 
Psychiatric Association 2000). Since PTSD is related to suicide (Amir, Kaplan, Efroni, \& Kotler, 1999) it is possible that nightmares act as a marker of trauma or PTSD and hence are related to suicide due to the trauma or PTSD.

Nightmares may also lead to suicide through cognitive deficits. Nightmares may wake a person during the night and make it difficult for him or her to return to sleep resulting in less sleep (Davis \& Wright, 2005) and possibly leading to sleep debt, which is the cumulative effect of sleep deprivation. Since reduced sleep is related to cognitive deficits (Stenuit \& Kerkhofs, 2008) and cognitive impairment is related to suicide attempts (Osvath, et al., 2005) it is possible that nightmares may also be related to suicide as they may result in difficulty sleeping.

In addition to being a cause, nightmares may also be a symptom of poor sleep quality. Since an individual must wake after a dream for the dream to be remembered, it is possible that both those with and without sleep problems have the same number of nightmares but those without sleep problems are not remembering them since they do not wake as often or for as long of periods during the night.

There have been a few studies that have investigated the relation between nightmares and suicidal behaviors. Cukrowicz et al. (2006) examined sleep disturbances in college students $(M=$ 19.2 years old) to see whether these disturbances were related to suicidal ideation. Utilizing a regression analysis, they found that disturbing dreams or nightmares and insomnia were related with increased risk of suicidal ideation. However, multiple regression revealed that nightmares, but not insomnia, were independently related with suicidal ideation.

Nightmares have also been examined in relation to suicide attempts. Sjostrom, Waern, and Hetta (2007) studied 165 patients age 18 to 68 years $(M=35.3$ years $)$ who had been admitted to the hospital following a medically serious suicide attempt. The most common sleep 
complaint was onset insomnia, although two-thirds of participants also reported having nightmares. A regression analysis revealed that onset insomnia, terminal insomnia, and nightmares were all significantly associated with higher scores of suicidality, defined as the risk of attempting suicide as measured by the Suicide Assessment Scale. Nightmares remained significantly related to high suicidality after controlling for DSM axis I disorders, posttraumatic stress disorder diagnoses, depression symptoms, and anxiety symptoms.

Although Sjostrom, Waern, and Hetta (2007) provide evidence that nightmares may be associated with suicide, there are limitations to their study. First, although all patients were interviewed within a week of admission, the self-report instruments used, including the questions on sleep, only assessed the last three days. It is likely that at least one if not more of these days were following the attempt and may be more indicative of the time following the attempt than before the attempt. Second, although Sjostrom, et al.(2007) demonstrated that nightmares were associated with high suicidality among people who are suicidal, the question of whether nightmares differentiate people who are suicidal from people who are not remains. Therefore, more research investigating the relation between nightmares and suicide is needed.

Taken together, these initial studies indicate that nightmares appear to be a promising risk factor for suicidal behaviors, but more research is needed, particularly to determine whether the relation between nightmares and suicidal ideation is explained by PTSD in a sample that is not limited to suicide attempters. Nightmares are not only highly co-morbid with PTSD, but are also a defining feature of PTSD, which has been shown to be related to suicide (Amir, Kaplan, Efroni, \& Kotler, 1999). Given that nightmares are so closely related to PTSD, it raises the question of whether they are associated with suicide independent of PTSD. Research demonstrating that nightmares distinguish between those with and without suicidal ideation 
would be an important step in determining whether nightmares can be isolated as a risk factor for suicide.

\section{Statement of the Problem}

Although $90 \%$ of those who die by suicide have a mental illness, the vast majority of those with a mental illness do not die by suicide. Therefore, there must be something other than presence of mental illness that distinguishes those who die by suicide from those who do not. There is promising research showing that insomnia and nightmares may both be related to suicidal ideation, but few studies have assessed whether these relations remain after controlling for related mental disorders such as depression, anxiety, and PTSD. Determining whether the effects of insomnia and nightmares explain suicidal thoughts and behaviors beyond mental disorders such as depression, anxiety, and PTSD is an important part of determining whether insomnia and nightmares should be considered risk-factors for suicide. Additionally, since insomnia symptoms may be influenced by nightmares, and vice versa, insomnia symptoms and nightmares must each be shown to be related to suicidal ideation independent of one another to be considered a risk-factor. If insomnia symptoms and nightmares are related to suicide independent of mental illness and independent of one another, they may help differentiate between those who die by suicide and those who do not.

\section{Present Study}

The present study aimed to characterize the relation between insomnia symptoms, nightmares, and suicidal ideation. The study tested whether insomnia symptoms and nightmares are statistical correlates of suicidal ideation. Further, it evaluated whether insomnia symptoms 
and nightmares are significantly related with suicidal ideation independent of one another and independent of symptoms of psychopathology.

Based on prior published findings, our first hypothesis was that insomnia symptoms would be significantly related to suicidal ideation. Similarly, our second hypothesis was that nightmares would be significantly related to suicidal ideation. The third hypothesis stated that nightmares would be related with suicidal ideation independent of insomnia symptoms. This hypothesis was based upon the work of Cukrowicz et al. (2006) showing that nightmares are associated with suicidal ideation independent of insomnia. Our fourth hypothesis predicted that nightmares would be significantly related with suicidal ideation when symptoms of insomnia, depression, anxiety, and PTSD were controlled. This hypothesis was based on the work of Sjostrom, Waern, and Hetta (2007), who found that nightmare frequency was related to severity of suicidality among individuals who had recently made a suicide attempt, independent of depression symptoms, anxiety symptoms, and PTSD diagnoses.

We also conducted exploratory analyses to evaluate whether there would be an interaction between nightmares and insomnia symptoms in relation to suicidal ideation. We predicted there would not be a significant interaction between nightmares and insomnia symptoms. This is an exploratory question that has not been tested in the previous literature. It is possible that having both nightmares and insomnia symptoms results in more suicidal ideation than having one or the other, but we predicted that there would not be a significant interaction because nightmares have completely explained the effect of insomnia symptoms on suicidal ideation in previous research (Cukrowicz et al. 2006). 
Method

\section{Participants}

The current study utilized data from a larger completed project, with data collected from November $6^{\text {th }}, 2008$ until December $8^{\text {th }}, 2008$, from undergraduate students enrolled at West Virginia University. The study was conducted in accordance with the IRB protocol. Participants consisted of 583 individuals, 451 females and 132 males,with an age range of 18-29 years (Mean age $=19.4$ years, $S D=1.7$ years). In keeping with the characteristics of the region, most participants $(96.1 \%)$ were Caucasian.

\section{Measures}

The Disturbing Dreams and Nightmare Severity Index (DDNSI; Krakow et al. 2002). The DDNSI is a revised version of the Nightmare Frequency Questionnaire (Krakow et al., 2000) and was used to measure nightmare severity and frequency for the last year prior to the attempt. It measures the number of nights with nightmares per week (0-7 nights) and number of total nightmares per week (0-14 nightmares). The DDNSI also measures the severity and intensity of the nightmare on a Likert-type scale ranging from no problem (0) to extremely severe problem (6) as well as how often nightmares result in awakenings ranging from never/rarely (0) to always (4). A score greater than 10 may indicate the presence of a nightmare disorder (Krakow et al., 2002). In the current sample the mean was $3.78(S D=4.96)$ with acceptable reliability $(a=0.87)$ The Center of Epidemiological Studies Depression Scale (CES-D; Radloff, 1977). The CES-D is a 20-item, self-report measure designed to assess levels of depression. The CES-D is scored on a four-point scale (0-3) with scores ranging from $0-60$ with 16 being a common cutoff for clinical referral. It has high internal consistency for both the general (alpha $=.85)$ and clinical (alpha $=.90)$ populations (Radloff, 1977). It has been demonstrated as a valid screening 
measure for detecting depressive symptoms (Weissman, Sholomskas, Pottenger, Prusoff, \& Locke, 1977). The CES-D was used in the current study as a measure of the severity of depressive symptoms. In the current sample the mean was $16.38(S D=9.88)$ with acceptable reliability $(a=0.90)$.

The Insomnia Severity Index (ISI; Bastien, Vallieres, \& Morin, 2001). The ISI is a 7-item self-report scale that assesses the individual's subjective report of insomnia for the past two weeks. Each item is scored on a 0-4 scale with total scores ranging from 0-28. Scores of 0-14 are considered to be sub-clinical insomnia or no insomnia, 15-21 are considered moderate insomnia, and 22-28 are considered to be severe insomnia. The ISI has been shown to have adequate testretest reliability over three months and concurrent validity with sleep diaries and polysomnography and has been used in previous research as a measure of insomnia severity (Bastien, Vallieres, \& Morin, 2001; Savard, Savard, Simard, \& Ivers, 2005) The ISI has also been used to determine the presence or absence of clinically significant insomnia symptoms using a cutoff of 15 (Bernert, Merrill, Braithwaite, Van Orden, Joiner, 2007; Tang, Wright, Salkovskis, 2007). In the current sample the mean was $8.84(S D=4.69)$ with acceptable reliability $(a=0.84)$

The PTSD Checklist-Civilian Version (PCL; Weathers, et al., 1993). The PCL is a measure of Posttraumatic stress disorder symptom severity. It consists of 17 questions in which participants are asked if they are bothered by each symptom on a scale ranging from 1 (not at all) to 5 (extremely), with total scores ranging from 17 to 85 . There is no single agreed-upon cutoff for the PCL, but 44 and 50 have each been used as cutoff scores in prior research (Ruggiero, Del Ben, Scotti, \& Rabalais, 2003). The PCL has high internal consistency (alpha $=0.94)$ and has been shown to be valid when compared to a clinician-administered PTSD scale (Peterlin, Tietjen, 
Meng, Lidicker, \& Bigal, 2008). In the current sample the mean was $34.30(S D=11.81)$ with acceptable reliability $(a=0.92)$

The Suicidal Behaviors Questionnaire (SBQ; Linehan, 1981). The SBQ, shortened by Cole (1988), is a 4-item, self-report measure designed to assess levels of suicidal ideation. The SBQ's four items are summed to create a score ranging from 0 to 16 . The first item inquires about past suicidal thoughts and suicide attempts, the second and third items assess past suicidal ideation and threats, and the fourth item asks about future suicidal behavior. It has moderate internal consistency with an alpha of 0.75 in a clinical sample and 0.80 in a non-clinical sample. It has been validated as a measure of suicidal ideation (Cotton, Peters, \& Range, 1995). The SBQ was used in the study as a measure of suicidal ideation. In the current sample the mean was 1.71 $(S D=2.74)$ with acceptable reliability $(a=0.84)$

The Zung Self-Rating Anxiety Scale (SAS; Zung, 1971). The SAS is a 20 item measure of anxiety symptoms. Participants are asked to mark the option that corresponds with how they have felt or behaved in the last several days. Each item is rated on a one to four scale with 1 being "A little of the time" and 4 being "Most of the time" (Zung, 1971). The total score ranges from 20 to 80 with scores of 50 or higher indicating clinically-significant anxiety symptoms (Zung, 1986). The SAS has been shown to discriminate between a normal adult sample and those with anxiety disorders. In the current sample the mean was $36.83(S D=8.31)$ with acceptable reliability $(a=0.85)$

\section{Procedure}

The current study utilized data from a larger completed project, with data collected from November $6^{\text {th }}, 2008$ until December $8^{\text {th }}, 2008$, from undergraduate students enrolled at West Virginia University. The study was approved by the Institutional Review Board of West Virginia 
University. Data were collected using the SONA online survey system. Participants logged onto the SONA system and selected the study. The participants were then asked to read a cover sheet informing them of the purpose of the project and their rights as a participant. Participants then completed the survey. At the conclusion of the survey participants were shown a referral sheet with contact information for local mental health services should they desire counseling following the study.

Analyses

Data were checked prior to conducting analyses. There were originally 614 participants in the dataset. All responses were viewed by undergraduate research assistants to identify any biased or inappropriate responding. Additionally, participants who took 9 minutes or less to complete the survey (duration $M=19.7, S D=10.4$ ) were reviewed a second time by the investigators.

There were 31 individuals removed from the dataset, resulting in data from 583 individuals being used for the analyses. One individual was removed because his reported age was lower than 18 , which is the required age to participate. Six individuals were removed due to having blank or mostly blank surveys. Fourteen individuals were removed due to apparently biased response set, indicated by blatant inconsistencies in responding (e.g. reporting never having had a nightmare and yet having a severe nightmare problem) or flippant responses. Lastly, the data from ten participants could not be used in the analyses due to having incomplete data on the SBQ4, which was the dependent variable for all of the analyses.

Missing data analyses were run on all scales used in the analyses. One-way ANOVAs were conducted to test whether the other scales differed based upon whether or not there were any items missing from that scale (missingness). Missingness of the SAS was significantly 
related with scores on the PCL, $F(1,576)=5.69, p<.05$, with those missing data on the SAS having lower scores on the PCL. Missingness on the SAS was not related with the other variables including the SBQ $F(1,582)=.00, p=.993$, which is the dependent variable for all of the analyses. Little's Missing Completely At Random (MCAR) test was conducted using all of the scales to be utilized in the analyses. Little's MCAR test was not significant, $\chi^{2}(59, N=490)=$ $64.38, p=.29$, indicating that the missing data from the SAS may be assumed to be missing at random (Tabachnick \& Fidell, 2007, pg. 63). Missingness on each of the other variables was unrelated to the dependent variable. Therefore, data missing from all of the measures can be assumed to be missing at random due to their missingness not being related to the dependent variable.

Given that all data can be assumed to be missing at random, it was appropriate to conduct multiple imputation. Missing scores for all of the independent variables were imputed using the CES-D, SAS, PCL, ISI, DDN, and SBQ4 for hypotheses one through four. Missing values were not imputed for the SBQ4 as it is the dependent variable in all of the analyses. Scores were not imputed for the exploratory question as multiple imputation is not appropriate for testing interactions. The data were imputed five times and then combined to yield the imputed results. $\mathrm{R}^{2}$ and standardized beta values were determined by averaging their values across the five imputations (von Hippel, 2009). In order to test the interaction between nightmares, symptoms of insomnia, and suicidal ideation, both nightmares and symptoms of insomnia were centered so their mean score was 0 .

Due to the high correlation between the symptoms of depression, anxiety, and PTSD, separate regression analyses were run with each of those variables and nightmares in relation to suicidal ideation. Results indicated that multicollinearity did not result in differences between 
nightmares and suicidal ideation. Additionally, all of the hypotheses were tested using both multiple imputation and listwise deletion and no significant differences were found between the two methods of handling missing data.

The analyses consisted of simple and multiple linear regressions and an ANOVA. Preliminary analyses were run to ensure normality, homoscedacity, and linearity. It was found that the Suicidal Behaviors Questionnaire significantly deviated from normality ( kurtosis $=5.4$ ). Since many of the values for the Suicidal Behaviors Questionnaire were 0, a square-root transformation was employed to restore normality. Following the transformation both the skew and kurtosis were less than $|1|$ (skew $=.87$, kurtosis $=-.28)$, indicating that the transformed data do not deviate significantly from normality.

An ANOVA was utilized to test for effects of sex. It was determined that sex was not significantly related to suicidal ideation and hence sex was not controlled for in the analyses.

\section{Results}

In order to test the first hypothesis, that insomnia symptoms will be significantly related to suicidal ideation, a simple linear regression was performed with insomnia symptoms as the independent variable and suicidal ideation as the dependent variable. Insomnia symptoms were significantly related to suicidal ideation, $\beta=.045$ ( $S E=.009), p<.01$. Insomnia symptoms explained a small but significant amount of the variance in suicidal ideation, $R^{2}=.05$. Therefore, the first hypothesis that insomnia symptoms would be significantly related with suicidal ideation was supported.

A simple linear regression was performed to test the second hypothesis that nightmares would be significantly related to suicidal ideation. Nightmares were the independent variable and suicidal ideation was the dependent variable in the regression. Nightmares were significantly 
related with suicidal ideation, $\beta=.054(S E=.008), p<.01$. Additionally, nightmares explained a significant amount of the variance in suicidal ideation $R^{2}=.07$. Therefore, the second hypothesis, that nightmares would be significantly related with suicidal ideation, was supported.

To test the third hypothesis, that nightmares would be related with suicidal ideation independent of insomnia symptoms, a multiple linear regression was utilized with insomnia symptoms being entered in step one and nightmares at step two (see Table 3). In step one, insomnia symptoms significantly predicted suicidal ideation, as shown above. In step two, both insomnia symptoms and nightmares were significantly associated with suicidal ideation. Step two explained a significant amount of variance in suicidal ideation $R^{2}=.098$, with an increase in $R^{2}$ of $.052, F(1,582)=33.7, p<.01$. Therefore, the third hypothesis, that nightmares would be related to suicidal ideation independent of insomnia symptoms, was supported.

In order to test the fourth hypothesis, that nightmares would be significantly related with suicidal ideation when symptoms of insomnia, depression, anxiety, and PTSD are controlled, a multiple linear regression was utilized (see Table 4). Depression symptoms, anxiety symptoms, and PTSD symptoms were added in the first step of the regression. The second step consisted of insomnia symptoms and the third step of the regression consisted of nightmares.

In step one, depression symptoms and PTSD symptoms were significantly related with suicidal ideation whereas anxiety symptoms were not related with suicidal ideation.

In step two, insomnia symptoms were added to the regression. Depression symptoms and PTSD symptoms remained significantly related with suicidal ideation. Insomnia symptoms were not significantly related to suicidal ideation.

Nightmares were added to the regression in step three. Depression symptoms, PTSD symptoms, and nightmares were significantly related with suicidal ideation whereas anxiety 
symptoms and insomnia symptoms were not related with suicidal ideation. The $R^{2}$ change between the second and third step was significant, $R^{2}$ change $=.009, F(1,582)=6.52, p<.05$. Therefore, the fourth hypothesis, that nightmares would be significantly related with suicidal ideation when depression symptoms, anxiety symptoms, and PTSD symptoms are controlled, was supported.

Lastly, a regression analysis was conducted to test for an interaction between insomnia symptoms and nightmares in relation to suicidal ideation. The interaction of insomnia symptoms and nightmares was not significant, $F(1,573)=.24, p=.63$.

\section{Discussion}

The results of this study indicate that both insomnia symptoms and nightmares are related to suicidal ideation independent of each other. However, when symptoms of depression, anxiety, and PTSD are controlled, nightmares, but not insomnia symptoms, are related with suicidal ideation. These findings suggest that nightmares may be a risk-factor for suicidal ideation beyond symptoms of insomnia, depression, anxiety, and PTSD.

Although suicide is a significant problem, it is also a low base-rate phenomenon making it difficult to predict who is in danger of dying by suicide. One way to determine one's risk of suicidal behavior is through assessing risk-factors. Although mental illness is a primary riskfactor and $90 \%$ of those who die by suicide have a mental illness, most individuals with a mental illness do not die by suicide. Therefore, there is a need for risk-factors that explain variance in suicide beyond mental illness. The purpose of this study was to lay the groundwork for determining whether insomnia symptoms or nightmares may be risk-factors for suicide by assessing their relation with suicidal ideation, independent of symptoms of depression, anxiety, and PTSD. 
The finding that nightmares are related to suicidal ideation after controlling for symptoms of insomnia, depression, anxiety, and PTSD is significant as it suggests that nightmares may be a risk-factor for suicidal ideation that can explain variance in suicidal ideation beyond symptoms of these three mental illnesses. It is possible that nightmares remain significant because they are not easily controlled and are unpredictable which may lead to hopelessness in those with nightmares. Additionally, the presence of nightmares may add distress above and beyond symptoms of insomnia, depression, anxiety, and PTSD and the additional distress may be resulting in higher suicidal ideation. More research is needed to determine why nightmares are independently related with suicidal ideation. The finding that nightmares are independently related with suicidal ideation is significant as it has implications for identification of those at risk of suicide as well as for treatment. The findings suggest that those who have nightmares and a mental illness may be at higher risk of suicidal ideation than those with only a mental illness. Although these findings are demonstrated for suicidal ideation, further research is needed to extend these findings to death by suicide. Since therapies for nightmares exist, this finding also presents an opportunity for intervention with those who may be at higher risk of suicidal ideation due to nightmares.

The current study found that insomnia symptoms were associated with suicidal ideation independent of nightmares. This finding contrasts with the findings of Cukrowicz, et al. (2006), who found that insomnia symptoms were not significantly related with suicidal ideation after controlling for nightmares. Nonetheless, it is possible that the null findings of Cukrowicz, et al. (2006) may have been due to the relatively small effect size of insomnia symptoms. Cukrowicz, et al. (2006) utilized a sample of 222 college students whereas the current study utilized 583 college students providing ample power to detect even small effect sizes. Although symptoms of 
insomnia were not significantly related with suicidal ideation after controlling for symptoms of depression, anxiety, and PTSD, they remain important due to their relation to mental disorders such as depression. Research has demonstrated that insomnia is not only a symptom but is also a risk-factor for both new onset and recurrence of Major Depressive Disorder (Perlis, et al. 2006). Even though insomnia symptoms may not explain suicidal ideation beyond symptoms of depression, anxiety, and PTSD, they may play a role in the development of depression, and hence be an important variable for suicide research. Additionally, the current study found that nightmares were related to suicidal ideation even after symptoms of insomnia, depression, anxiety, and PTSD were controlled. This finding is similar to the findings of Sjostrom, Waern, and Hetta (2007) who found that nightmares remained significantly associated with high suicidality in a sample of suicide attempters after controlling for DSM axis I disorders that included PTSD, anxiety, and depression. The current findings expand upon this research by showing that nightmares are associated with suicidal ideation after controlling symptoms of insomnia, depression, anxiety, and PTSD in a college student population. This expands upon the findings of Sjostrom, Waern, and Hetta (2007) by suggesting that nightmares may be associated with suicide in a population that is not restricted to suicide attempters.

\section{Limitations}

There are several limitations of the current study. First, the use of a college population is a limitation as suicidal behavior differs by age and education level. The population in this study was between 18 and 29, was mostly female, and had at least a high school education. Therefore, these findings may not generalize to other populations. Nonetheless, it is an important population to study as suicide is the third leading cause of death for college-age individuals (Heron et al., 2009). 
Second, since the SBQ is a measure of lifetime suicidal ideation, it may be less sensitive to recent changes in suicidal ideation. Nevertheless, the fact that we measured current nightmares and lifetime SBQ would most likely reduce the relation between the measures and conservatively bias outcomes, only strengthening the findings. Further, the SBQ is a commonly used, validated measure of suicidal ideation making it appropriate for this study.

Third, using symptoms of disorders instead of diagnosing disorders can be viewed as a limitation of the current study. However, the current study employed validated instruments that have been shown to be highly related with diagnoses of those disorders.

The use of suicidal ideation instead of suicidal attempts or death by suicide is a limitation of this study. Although suicidal ideation is one of the strongest predictors of suicidal behavior, a low percentage of individuals with suicidal ideation go on to die by suicide. However, suicidal ideation is highly correlated with suicide attempts and death by suicide and is one of the strongest predictors of death by suicide. For this reason it is often used in suicide research instead of suicide attempts or death by suicide.

Lastly, the use of a cross-sectional design is a limitation of this study as it is impossible to determine whether insomnia symptoms or nightmares predict changes in suicidal ideation or changes in suicidal ideation predict changes in sleep. Nonetheless, the finding that insomnia symptoms and nightmares are related to suicidal ideation is still valid. Further, the finding that nightmares are related with suicidal ideation after controlling for symptoms of insomnia, depression, anxiety, and PTSD still suggests that nightmares may be a risk-factor for suicide.

\section{Conclusions and Future Directions}

The goal of the current research was to lay the groundwork to determine whether insomnia symptoms or nightmares may be risk-factors for suicide. Although this study shows 
that nightmares are related to suicidal ideation, much work is left to be done to determine the relation between nightmares and suicide. Future studies should examine the relation between insomnia symptoms, nightmares, and suicidal ideation in different age groups, especially older adults, to determine whether these findings generalize beyond a college-age sample. Prospective research would also be valuable to determine whether changes in insomnia symptoms or nightmares are related to changes in suicidal ideation. Although measuring symptoms of disorders, as done in the current study, may be a good proxy for the disorder, future research utilizing diagnoses should be conducted. Using diagnoses instead of symptoms will increase the validity of the study and will shed light on whether having the disorder, instead of just having symptoms of the disorder, results in differences. Similarly, research utilizing medically-serious suicide attempts or death by suicide instead of suicidal ideation is needed to determine whether nightmares are a risk-factor for suicide.

Although there are limitations, the current study is one of the first studies demonstrating that nightmares are associated with insomnia symptoms while controlling for symptoms of depression, anxiety, and post-traumatic stress disorder. Combined with the findings of Sjostrom et al., 2007, the current study demonstrates the need to further investigate the relation between nightmares and suicidal ideation, medically-serious suicide attempts, and death by suicide. Through further investigation, it can be determined whether nightmares are a risk-factor for suicide. If so, this knowledge may change the way suicidal individuals are accessed and treated. 


\section{References}

American Psychiatric Association (2000). Diagnostic and statistical manual of mental Disorders (4 $4^{\text {th }}$ ed.tr). Washington, DC: Author.

Amir, M., Kaplan, Z., Efroni, R., \& Kotler, M. 1999. Suicide risk and coping styles in posttraumatic stress disorder patients. Psychotherapy and Psychosomatics, 68(2), 76-81.

Barbe, R.P., Williamson, D.E., Bridge, J.A., Birmaher, B., Dahl, R. E., Axelson, D.A., et al. (2005).Clinical differences between suicidal and nonsuicidal depressed children and adolescents. Journal of Clinical Psychiatry, 66, 492-498.

Bastien, C.H., Vallieres, A., \& Morin, C.M., (2001). Validation of the insomnia severity index as an outcome measure for insomnia research. Sleep Medicine, 2(4), 297-307.

Bernert, R. A., Merrill, K. A., Braithwaite, S. R., Van Orden, K. A., \& Joiner, T. E., (2007). Family life stress and insomnia symptoms in a prospective evaluation of young adults. Journal of Family Psychology, 21(1), 58-66.

Cole, D.A. (1988). Hopelessness, social desirability, depression, and parasuicide in two college student samples. Journal of Consulting and Clinical Psychology, 56, 131-136.

Cotton, C. R., Peters, D. K., \& Range, L. M. (1995). Psychometric properties of the suicidal behaviors questionnaire. Death Studies, 19, 391-397.

Cukrowicz, K.C., Otamendi, A., Pinto, J.V., Bernert, R.A., Krakow, B., \& Joiner, T.E. (2006). The impact of insomnia and sleep disturbances on depression and suicidality. Dreaming, 16(1), 1-10.

Davis. J. L., \& Wright, D. C.(2005). Case series utilizing exposure, relaxation, and rescripting therapy: impact on nightmares, sleep quality, and psychological distress. Behavioral Sleep Medicine, 3(3), 151-157. 
Fawcett, J., Scheftner, W.A., Fogg, L., \& Clark, D.C. (1990). Time-related predictors of suicide in major affective disorder. American Journal of Psychiatry, 147(9), 1189-1194.

Fujino, Y., Mizoue, T., Tokui, N., \& Yoshimura, T. 2005. Prospective cohort study of stress, life satisfaction, self-rated health, insomnia, and suicide death in Japan. Suicide and Life Threatening Behavior, 35(2), 227-237.

Hall, R.C.W., Platt, D.E., \& Hall, R.C.W. (1999). Suicide risk assessment: a review of risk factors for suicide in 100 patients who made severe suicide attempts. Evaluation of suicide risk in a time of managed care. Psychosomatics, 40(1), 18-27.

Heron, M., Hoyert, D.L., Murphy, S.L., Xu, J., Kochanek, K.D., \& Tejada-Vera, B. (2009). Deaths: final data for 2006. National Vital Statistics Reports, 57(14). Retrieved July 28, 2009 from http://www.cdc.gov/nchs/data/nvsr/nvsr57/nvsr57_12.pdf.

Koffel, E., \& Watson, D. (2009). The two-factor structure of sleep complaints and its relation to depression and anxiety. Journal of Abnormal Psychology, 118, 183-194.

Krakow, B., Hollifield, M., Schrader, R., Koss, M., Tandberg, D., Lauriello, J., et al. (2000). A controlled study of imagery rehearsal for chronic nightmares in sexual assult survivors with PTSD: A preliminary report. Journal of Traumatic Stress, 13, 589-609.

Krakow, B.J., Melendrez, D. C., Johnston, L. G., Clark, J. O., Santana, E. M., Warner, T. D, et al. (2002). Sleep dynamic therapy for Cerro Grande Fire Evacuees with posttraumatic stress symptoms: A preliminary report. Journal of Clinical Psychiatry, 63(8), 673-684.

McGirr, A., Renaud, J., Seguin, M., Alda, M., Benkelfat, C., Lesage, A., et al. (2007). An examination of DSM-IV depressive symptoms and risk for suicide completion in major depressive disorder: A psychological autopsy study. Journal of Affective Disorders, 97(13), 203-209. 
Osvath, P., Kovacs, A., Voros, V., \& Fekete, S. (2005) Risk factors of attempted suicide in the elderly: the role of cognitive impairment. International Journal of Psychiatry in Clinical Practice, 9(3), 221-225.

Perlis, M. L., Smith, L.J., Lyness, J.M., Matteson, S.R., Pigeon, W.R., Jungquist, C.R., et al. (2006). Insomnia as a risk factor for onset of depression in the elderly. Behavioral Sleep Medicine, 4(2), 104-113.

Peterlin, B. L., Tietjen, G., Meng, S., Lidlicker, J., \& Bigal, M. (2008). Post-traumatic stress disorder in episodic and chronic migraine. Headache, 48, 517-522.

Radloff, L. (1977). The CES-D Scale: A self-report depression scale for research in the general population. Applied Psychological Measurement, 1(3), 385-401.

Ruggiero, K.J., Del Ben, K., Scotti, J. R., \& Rabalais, A. E. (2003). Psychometric properties of the PTSD Checklist-Civilian Version. Journal of Traumatic Stress, 16(5), 495-502.

Savard, M.H., Savard, J., Simard, S., \& Ivers, H., 2005. Empirical validation of the insomniaseverity index in cancer patients. Psycho-Oncology, 14, 429-441.

Sjostrom, N., Waern, M., \& Hetta, J. 2007. Nightmares and sleep disturbances in relation to suicidality in suicide attempters. SLEEP, 30(1), 91-95.

Stenuit, P., \& Kerkhofs, M., 2008. Effects of sleep restriction on cognition in women. Biological Psychology, 77(1), 81-88.

Tabachnick, B. G., \& Fidell, L. S. (2007). Using multivariate statistics ( $5^{\text {th }}$ ed.). Boston: Pearson Education

Tang, N. K. Y., Wright, K. J., \& Salkovskis, P. M., (2007). Prevalence and correlates of clinical insomnia co-occuring with chronic back pain. Journal of Sleep Research, 16, 85-95. 
Tidemalm, D., Langstrom, N., Lichtenstein, P., \& Runeson, B. (2008). Risk of suicide after suicide attempt according to coexisting psychiatric disorder: swedish cohort study with long term follow-up. British Medical Journal, 337, a2205.

von Hippel, P.T. (In Press). How to impute squares, interactions, and other transformed variables. Sociological Methodology.

Weathers, F. W., Litz, B.T., Herman, D.S., Huska, J.A., \& Keane, T. M. (1993, October). The PTSD checklist (pcl); reliability, validity, and diagnostic utility. Paper presented at The International Society for Traumatic Stress Studies, San Antonio, TX.

Weissman, M. M., Sholomskas, D., Pottenger, M., Prusoff, B. A., \& Locke, B. Z. (1977) Assessing depressive symptoms in five psychiatric populations: a validation study. American Journal of Epidemiology, 106(3), 203-214.

Wood, J.M., Bootzin, R. R., Rosenhan, D., Nolen-Hoeksema, S., \& Jourden, F. (1992). Effects of the 1989 san francisco earthquake on frequency and content of nightmares.Journal of Abnormal Psychology, 101(2), 219-224.

Zung, W. W. (1971). A rating instrument for anxiety disorders. Psychosomatics: Journal of Consultation Liaison Psychiatry, 12(6), 371-379.

Zung, W. W. (1986). Prevalence of clinically significant anxiety in a family practice setting. The American Journal of Psychiatry, 143(11), 1471. 
Table 1:

Descriptive statistics and the proportion of the sample over the clinical cutoff before imputation

\begin{tabular}{lccccc}
\hline \multicolumn{1}{c}{ Measure } & $\mathrm{N}$ & Mean & $S D$ & Clinical cutoff & \% over clinical cutoff \\
\hline Suicidal Ideation & 583 & 0.83 & 1.01 & N/A & N/A \\
Nightmares & 579 & 3.78 & 4.97 & 11 & $10.2 \%$ \\
Insomnia Symptoms & 581 & 8.87 & 4.70 & 15 & $13.1 \%$ \\
Depression Symptoms & 571 & 16.38 & 9.92 & 16 & $44.4 \%$ \\
Anxiety Symptoms & 490 & 36.82 & 8.31 & 50 & $7.8 \%$ \\
PTSD Symptoms & 577 & 34.33 & 11.82 & 50 & $12.1 \%$ \\
\hline
\end{tabular}


Table 2:

Correlations of independent and dependent variables

\begin{tabular}{lcccccc}
\hline Variable & 1 & 2 & 3 & 4 & 5 & 6 \\
& & & & & & \\
\hline 1. Suicidal Ideation & - & .27 & .21 & .41 & .37 & .44 \\
2. Nightmares & & - & .21 & .28 & .37 & .42 \\
3. Insomnia Symptoms & & & - & .53 & .60 & .53 \\
4. Depression Symptoms & & & - & .79 & .68 \\
5. Anxiety Symptoms & & & & - & .74 \\
6. PTSD Symptoms & & & & & & \\
\end{tabular}


Table 3:

Results for hypothesis 3: Multiple regression with insomnia symptoms in the first step and nightmares in the second step in relation to suicidal ideation.

\begin{tabular}{lccccc}
\hline Predictors & $R^{2}$ & $\beta$ & $S E$ & $t$ & $p$ \\
\hline Step 1: & .045 & & & & $<.01$ \\
Insomnia & & .045 & .009 & 5.23 & $<.01$ \\
Step 2: & .097 & & & & $<.01$ \\
Insomnia & & .035 & .009 & 4.07 & $<.01$ \\
Nightmares & & .047 & .008 & 5.80 & $<.01$ \\
\hline
\end{tabular}


Table 4:

Results for hypothesis 4: Multiple regression with symptoms of depression, anxiety, and PTSD in the first step, insomnia symptoms in the second step, and nightmares in the third step in relation to suicidal ideation.

\begin{tabular}{|c|c|c|c|c|c|}
\hline Predictors & $R^{2}$ & $\beta$ & $S E$ & $t$ & $p$ \\
\hline Step 1: & .217 & & & & \\
\hline Depression & & .024 & .007 & 3.63 & $<.01$ \\
\hline Anxiety & & -.006 & .009 & -0.62 & .54 \\
\hline PTSD & & .027 & .005 & 5.49 & $<.01$ \\
\hline Step 2: & .221 & & & & \\
\hline Depression & & .025 & .007 & 3.77 & $<.01$ \\
\hline Anxiety & & -.002 & .009 & -0.20 & .84 \\
\hline PTSD & & .028 & .005 & 5.74 & $<.01$ \\
\hline Insomnia & & -.018 & .010 & -1.78 & .076 \\
\hline Step 3: & .230 & & & & \\
\hline Depression & & .026 & .007 & 3.94 & $<.01$ \\
\hline Anxiety & & -.005 & .010 & -0.53 & .60 \\
\hline PTSD & & .025 & .005 & 5.00 & $<.01$ \\
\hline Insomnia & & -.016 & .010 & -1.63 & .10 \\
\hline Nightmares & & .021 & .008 & 2.53 & .01 \\
\hline
\end{tabular}


Appendix 1: The Insomnia Severity Index

Insomnia Severity Index (ISI)

1. Please rate the current (i.e. last 2 weeks) SEVERITY of your insomnia problem(s).

Difficulty Falling

Asleep:

Difficulty Staying

Asleep:

Problem waking too

early:
None

0

0

0
Mild

1

1

1
Moderate

2

2

2
Severe

3

3

3

2. How SATISFIED/dissatisfied are you with your current sleep pattern?

Very Satisfied

Very Dissatisfied

0

1

2

3

4

3. To what extent do you consider your sleep problem to INTERFERE with your daily functioning (e.g. daytime fatigue, ability to function at work/daily chores, concentration, memory, mood, etc.)

\begin{tabular}{|c|c|c|c|c|}
\hline $\begin{array}{l}\text { Not at all } \\
\text { Interfering }\end{array}$ & A Little & Somewhat & Much & $\begin{array}{l}\text { Very Much } \\
\text { Interfering }\end{array}$ \\
\hline
\end{tabular}

$\begin{array}{lllll}0 & 1 & 2 & 3 & 4\end{array}$

4. How NOTICABLE to others do you think your sleeping problem is in terms of impairing the quality of your life?

Not at all

A Little Somewhat

Much

Very Much

Interfering

Interfering

0

1

2

3

4

5. How WORRIED/distressed are you about your current sleep problem?

Not at all

0
A Little

Somewhat
Much

Very Much 
Appendix 2: The Disturbing Dreams and Nightmares Severity Index

1. How often did you have disturbing dreams and/or nightmares: (Circle one, then follow the arrow)

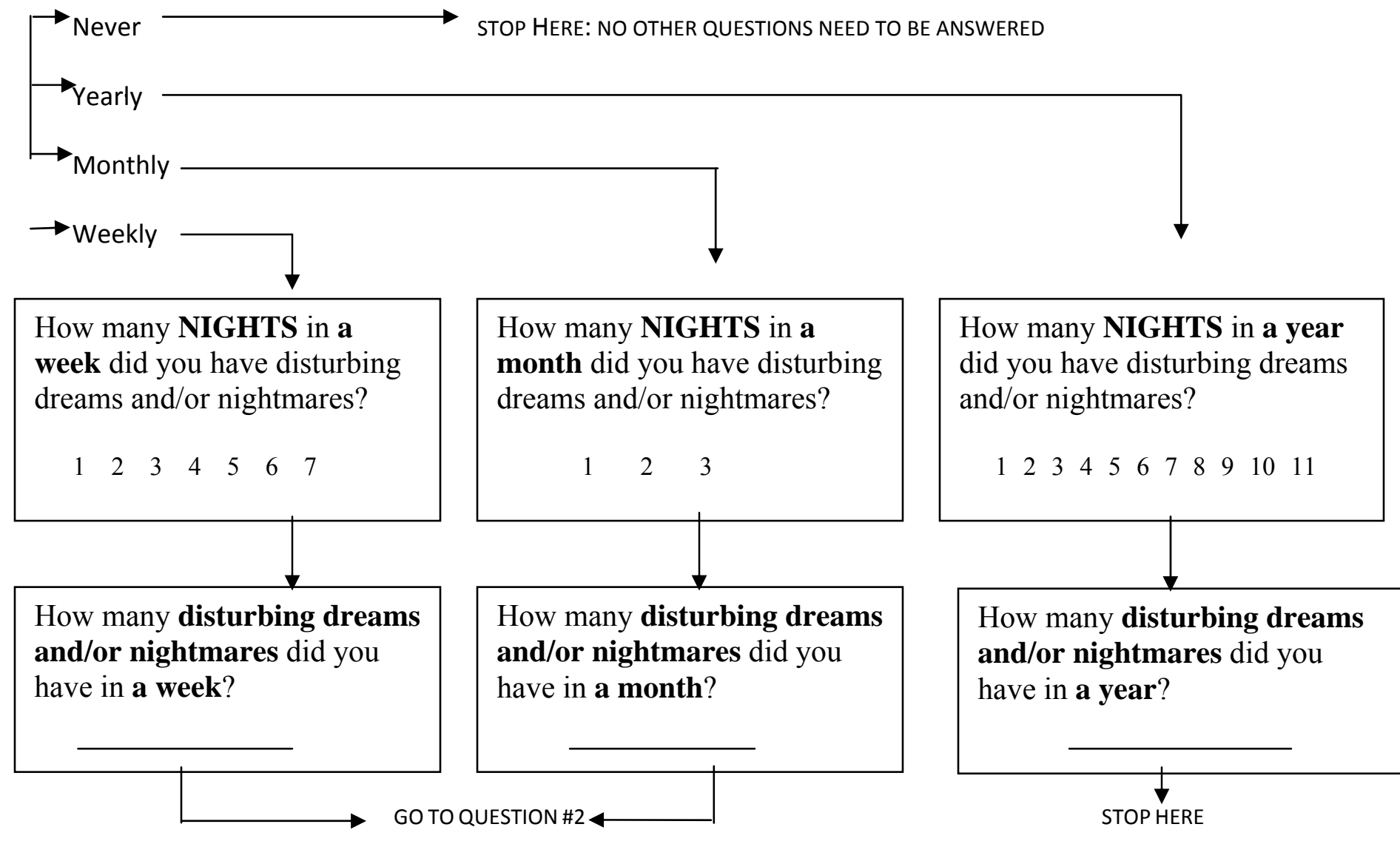

2. Please estimate the NUMBER of months or years you have had disturbing dreams and/or nightmares:

months years

3. On average, do your nightmares wake you up? (Circle answer)

$$
\text { Never/Rarely Occasionally Sometimes Frequently Always }
$$

4.How would you rate the SEVERITY of your disturbing dreams and/or nightmare problem? (Circle answer)

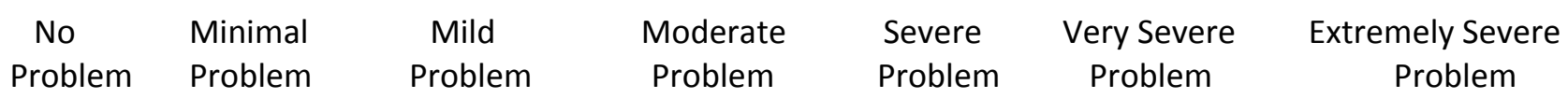

5. How would you rate the INTENSITY of your disturbing dreams and/or nightmares? (Circle answer)

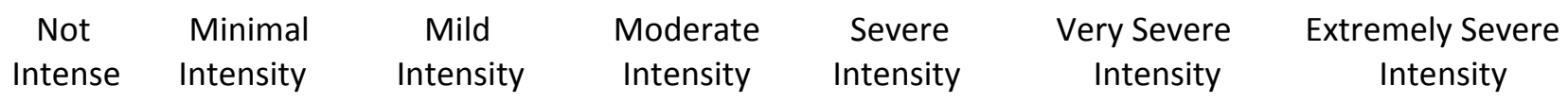




\section{Appendix 3: The Center for Epidemiologic Studies Depression Scale}

Below is a list of ways you might have felt or behaved. Please tell me how often you have felt this way during the past week.

\begin{tabular}{|c|c|c|c|c|}
\hline \multirow[b]{2}{*}{ DURING THE PAST WEEK: } & \multicolumn{4}{|c|}{ Last Week } \\
\hline & $\begin{array}{l}\text { Not at All } \\
\text { or Less } \\
\text { Than } 1 \\
\text { Day }\end{array}$ & 1-2 Days & 3-4 Days & 5-7 Days \\
\hline \multicolumn{5}{|c|}{$\begin{array}{l}\text { 1. I was bothered by things that } \\
\text { usually don't bother me. }\end{array}$} \\
\hline \multicolumn{5}{|c|}{$\begin{array}{l}\text { 2. I did not feel like eating; my } \\
\text { appetite was poor. }\end{array}$} \\
\hline \multicolumn{5}{|c|}{$\begin{array}{l}\text { 3. I felt that I could not shake off } \\
\text { the blues even with help from } \\
\text { my family or friends. }\end{array}$} \\
\hline \multicolumn{5}{|l|}{$\begin{array}{l}\text { 4. I felt I was just as good as } \\
\text { other people. }\end{array}$} \\
\hline \multicolumn{5}{|c|}{$\begin{array}{l}\text { 5. I had trouble keeping my mind } \\
\text { on what I was doing. }\end{array}$} \\
\hline \multicolumn{5}{|l|}{ 6. I felt depressed. } \\
\hline \multicolumn{5}{|c|}{$\begin{array}{l}\text { 7. I felt that everything I did was } \\
\text { an effort. }\end{array}$} \\
\hline \multicolumn{5}{|c|}{ 8. I felt hopeful about the future. } \\
\hline \multicolumn{5}{|c|}{$\begin{array}{l}\text { 9. I thought my life had been a } \\
\text { failure. }\end{array}$} \\
\hline \multicolumn{5}{|l|}{ 10. I felt fearful. } \\
\hline \multicolumn{5}{|l|}{ 11. My sleep was restless. } \\
\hline \multicolumn{5}{|l|}{ 12. I was happy. } \\
\hline \multicolumn{5}{|l|}{ 13. I talked less than usual. } \\
\hline \multicolumn{5}{|l|}{ 14. I felt lonely. } \\
\hline \multicolumn{5}{|l|}{ 15. People were unfriendly. } \\
\hline \multicolumn{5}{|l|}{ 16. I enjoyed life. } \\
\hline \multicolumn{5}{|l|}{ 17. I had crying spells. } \\
\hline 18. I felt sad. & & & & \\
\hline
\end{tabular}




\begin{tabular}{|l|l|l|l|l|}
\hline 19. I felt that people dislike me. & & & & \\
\hline 20. I could not get "going". & & & & \\
\hline
\end{tabular}




\section{Appendix 4: The Zung Self-Administered Anxiety Scale}

For each item below, please mark the option which best describes how often you felt or behaved this way during the past several days.

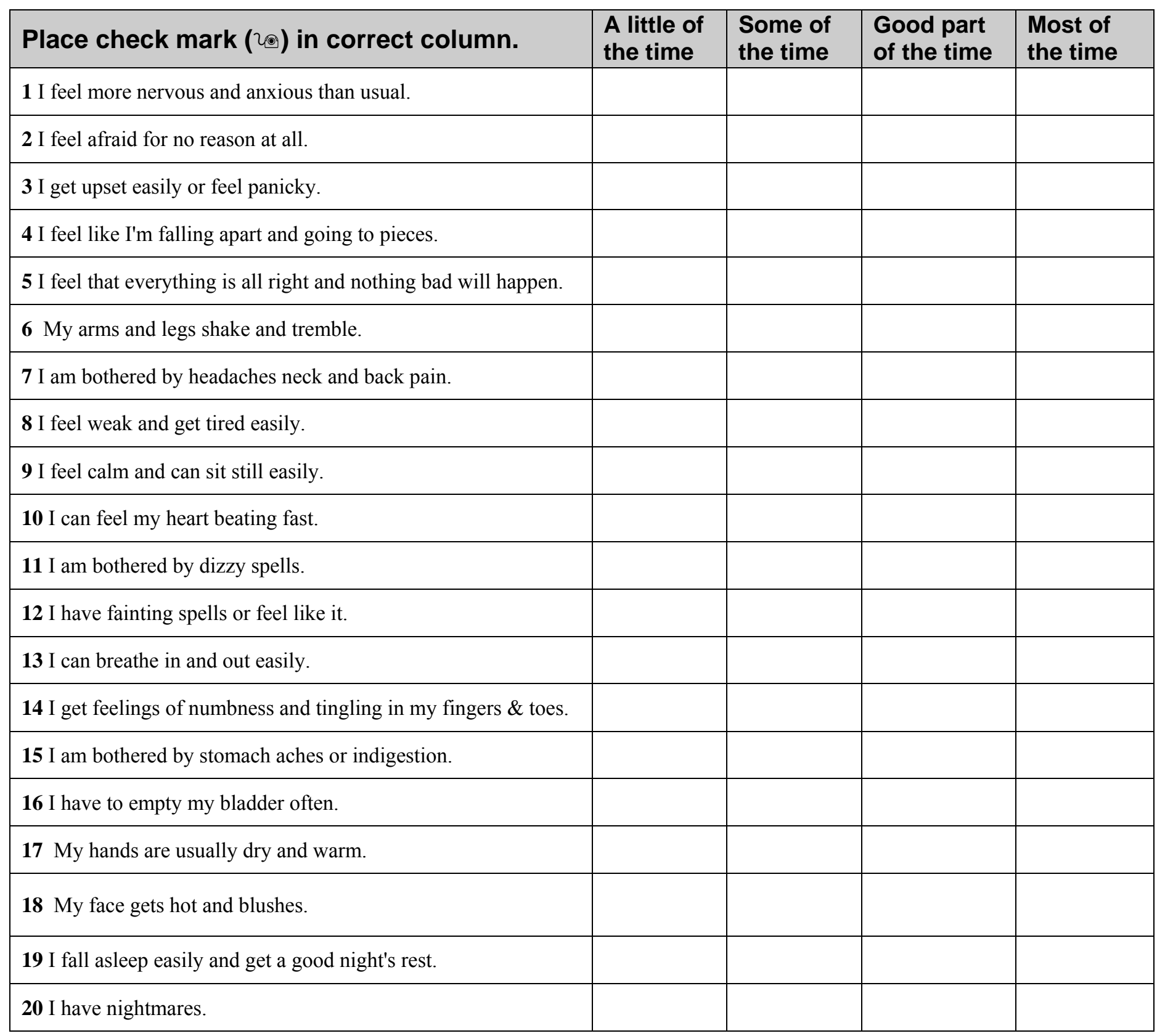




\section{Appendix 5: The PTSD Checklist Civilian Version}

INSTRUCTIONS TO PARTICIPANT: Below is a list of problems and complaints that people sometimes have in response to stressful experiences. Please read each one carefully, select the best option to indicate how much you have been bothered by that problem in the past month.

1. Repeated, disturbing memories, thoughts, or images of a stressful experience?

1. Not at all 2. A little bit 3. Moderately 4. Quite a bit 5. Extremely

2. Repeated, disturbing dreams of a stressful experience?

1. Not at all 2. A little bit 3. Moderately 4. Quite a bit 5. Extremely

3. Suddenly acting or feeling as if a stressful experience were happening again (as if you were reliving it)?

1. Not at all 2. A little bit 3. Moderately 4. Quite a bit 5. Extremely

4. Feeling very upset when something reminded you of a stressful experience?

1. Not at all 2. A little bit 3. Moderately 4. Quite a bit 5. Extremely

5. Having physical reactions (e.g., heart pounding, trouble breathing, sweating) when something reminded you of a stressful experience?

1. Not at all 2. A little bit 3. Moderately 4. Quite a bit 5. Extremely

6. Avoiding thinking about or talking about a stressful experience or avoiding having feelings related to it?

1. Not at all 2. A little bit 3. Moderately 4. Quite a bit 5. Extremely

7. Avoiding activities or situations because they reminded you of a stressful experience?

1. Not at all 2. A little bit 3. Moderately 4. Quite a bit 5. Extremely

8. Trouble remembering important parts of a stressful experience?

1. Not at all 2. A little bit 3. Moderately 4. Quite a bit 5. Extremely

9. Loss of interest in activities that you used to enjoy?

1. Not at all 2. A little bit 3. Moderately 4. Quite a bit 5. Extremely 
10. Feeling distant or cut off from other people?

1. Not at all 2. A little bit 3. Moderately 4. Quite a bit 5. Extremely

11. Feeling emotionally numb or being unable to have loving feelings for those close to you?

1. Not at all 2. A little bit 3. Moderately 4. Quite a bit 5. Extremely

12. Feeling as if your future will somehow be cut short?

1. Not at all 2. A little bit 3. Moderately 4. Quite a bit 5. Extremely

13. Trouble falling or staying asleep?

1. Not at all 2. A little bit 3. Moderately 4. Quite a bit 5. Extremely

14. Feeling irritable or having angry outbursts?

1. Not at all 2. A little bit 3. Moderately 4. Quite a bit 5. Extremely

15. Having difficulty concentrating?

1. Not at all 2. A little bit 3. Moderately 4. Quite a bit 5. Extremely

16. Being "super-alert" or watchful or on guard?

1. Not at all 2. A little bit 3. Moderately 4. Quite a bit 5. Extremely

17. Feeling jumpy or easily startled?

1. Not at all 2. A little bit 3. Moderately 4. Quite a bit 5. Extremely 


\section{Appendix 6: The Suicidal Behaviors Questionnaire}

In the previous sections, we have asked about some of your general thoughts and behaviors. The following questions ask about a specific topic- whether you have ever thought about suicide. Many people have had thoughts about suicide. Please answer honestly. Your responses will be kept confidential.

NOTE: A list of mental health agencies that can help individuals having thoughts of suicide is included at the end of the survey.

1._Have you ever thought about or attempted to kill yourself in your lifetime?

$0=$ No

$1=$ It was just a passing thought.

$2=$ I briefly considered it, but not seriously.

$3=$ I thought about it and was somewhat serious.

$4=$ I had a plan for killing myself which I thought would work and seriously considered it.

$5=$ I attempted to kill myself, but I do not think I really meant to die.

$6=$ I attempted to kill myself, and I really hoped to die.

3. How often have you thought about killing yourself in the last year?

$0=$ Not at all

$1=$ Rarely

$2=$ Sometimes

$3=$ Often

$4=$ Very often

4. Have you ever told someone that you were going to commit suicide, or that you might do it in your lifetime?

$0=$ No

$1=$ Yes, during one short period of time.

$2=$ Yes, during more than one period of time.

8. _ How likely is it that you will attempt suicide in your lifetime?

0

No Chance at all
1

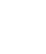

$\stackrel{2}{\text { Some Chance }}$
4

Very Likely

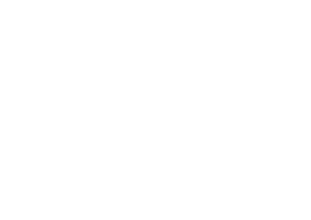

